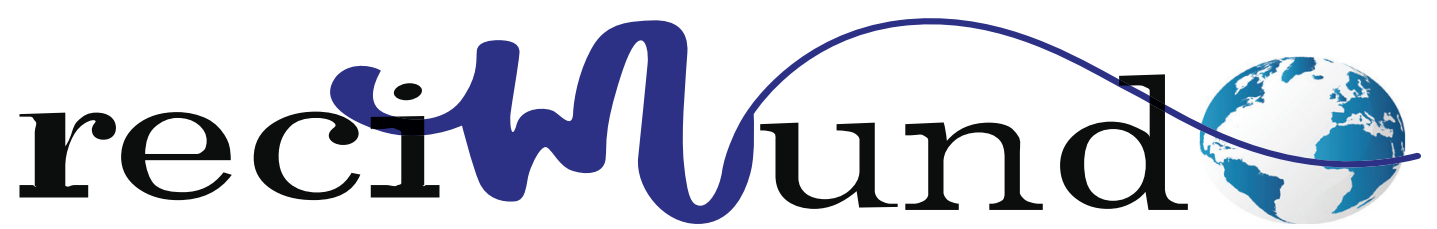

Revista Científica Mundo de la Investigación y el Conocimiento

DOI: 10.26820/recimundo/4.(4).noviembre.2020.236-248

URL: http://recimundo.com/index.php/es/article/view/943

EDITORIAL: Saberes del Conocimiento

REVISTA: RECIMUNDO

ISSN: 2588-073X

TIPO DE INVESTIGACIÓN: Artículo de revisión

CóDIGO UNESCO: 32 Ciencias Médicas

PAGINAS: $236-248$

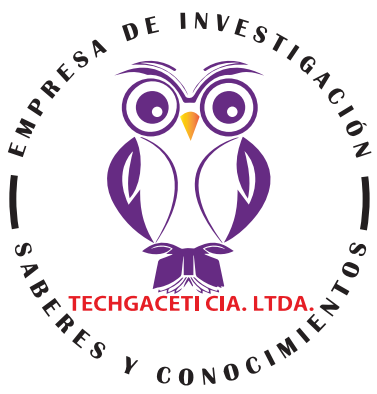

\title{
Conocimiento y uso de métodos anticonceptivos en estudiantes universitarios de medicina y enfermería, 2020
}

Contraceptive knowledge and the use in university medical and nursing students, 2020

Conhecimento anticoncepcional e 0 uso em estudantes universitários de medicina e enfermagem, 2020

Sara Elizabeth Bravo Salinas'; Diana Laura Guerra Ortega';

Vilma Amanda Uguña Rosas;3; Alex Alberto Castillo Zhizhpón ${ }^{4}$

RECIBIDO: 19/08/2020 ACEPTADO: 02/09/2020 PUBLICADO: 10/11/2020

1. Master en Atención de pacientes con VIH; Médica Especialista en Medicina Familiar; Médica General; Docente de la Universidad Católica de Cuenca, Carrera de Medicina; Cuenca, Ecuador; sara.bravo@ucacue.edu.ec; org/0000-0003-4878-1662

2. Médica Especialista en Medicina Familiar y Comunitaria; Médica General; Docente de la Universidad Católica de Cuenca, Carrera de Medicina; Cuenca, Ecuador; diana.guerra@ucacue.edu.ec; (iD https://orcid.org/0000-0001-5401-1061

3. Magister en Ciencias y Tecnologías Cosméticas; Magister en Docencia Universitaria; Diploma Superior en Investigación socio educativa título propio; Doctora Bioquímica Farmacéutica en las Áreas Clínica, Farmacéutica y Bromatológica; Docente de la Universidad católica de Cuenca, Carrera de Medicina; Cuenca, Ecuador; vilma.uguna@ucacue.edu.ec; (iD https:// orcid.org/0000-0002-3742-4080

4. Master en Atención de pacientes con VIH; Médica Especialista en Medicina Familiar; Médico General; Médico tratante del IESS; Azogues, Ecuador; acz8@hotmail.com; iD https://orcid.org/0000-0002-6487-7552

\section{CORRESPONDENCIA}

Sara Elizabeth Bravo Salinas

sara.bravo@ucacue.edu.ec

Cuenca, Ecuador 


\section{RESUMEN}

Introducción: El uso de métodos anticonceptivos depende del conocimiento de los mismos, para un uso responsable que permitan una vida sexual saludable. Objetivo: Determinar la relación del nivel de conocimiento y uso de métodos anticonceptivos en estudiantes Universitarios. Materiales y métodos: Estudio descriptivo correlacional de corte transversal, con población de estudio 477 estudiantes universitarios, se aplicó una encuesta, analizada mediante Razón de Prevalencias, OR e IC95\% calculado por distribución binomial, con interacción entre las variables mediante SPSS V20. Resultados: La edad de los participantes entre 17 y 27 años, el 54,7\% son mujeres, 75,3\% residen en Cañar y el $60 \%$ son sexualmente activos, de los cuales el 92,7\% manifestó conocer sobre métodos anticonceptivos, el 39,2\% no utiliza ningún método como medida de protección, el método preferido es el preservativo (32,5\%). Se demostró que existe $16,1 \%$ de estudiantes que manifestaron no asumir su responsabilidad ante un embarazo no deseado y de éstos el $61,1 \%$ son de sexo femenino, los estudiantes que no utilizan métodos anticonceptivos, son católicos en un 32\% (RP 1,32; IC95\% 1,122,63 ) y tienen el 50\% (RP 1,50; IC95\% 1,07-2,11) más probabilidades de contraer enfermedades de transmisión sexual, los que carecen de conocimientos tienen 2,4 veces (RP 2,41; IC95\% 1,90-3,06) más probabilidad de no usar ningún método. Conclusiones: El nivel de conocimiento es alto, pero el uso de anticonceptivos se encuentra por debajo de lo esperado, el ser católico y no conocer sobre anticonceptivos aumenta el riesgo de no usarlos, incrementando el riesgo de contraer enfermedades de transmisión sexual.

Palabras clave: Conocimiento, familia, métodos anticonceptivos.

\section{ABSTRACT}

Introduction: The responsible use of contraceptives that allow us to having a healthy sexual life, depends on the knowledge that we have of them. Tools and methods: Cross-section correlational descriptive study with a population of 477 college(university) students. A questionnaire was applied and analyzed throughout prevalence ratio, OR e IC95\% calculated by binomial distribution with interactions between the variables SPSSV20. Results: The age of the participants between 17 and 27 years of age,the $54.7 \%$ are women,75.3\% reside in Canar and the $60 \%$ are sexually active,from which $92.7 \%$ affirmed to know about the use of contraceptives, the $39.2 \%$ does not use any protective method. The preferred method is the Condon(32.5\%). It was demonstrated that $16.1 \%$ of the students have manifested not taking any responsibility on an oncoming, not desire,pregnancy. And among these the $61.1 \%$ are females. The students that not use contraceptives are Catholics on a 32\%(RP 1.32; IC95\% 1.12-2.63) and have the 50\%(RP 1.50;IC95\% 1.07-2.11) more probabilities of catching sexual transmited diseases. Those with lack of knowledge have 2.4 times(RP 2.41;IC95\% 1.30-3.06) more probabilities of not using any method. Conclusions: The grade of knowledge is high, but the use of contraceptives is way below expectations.Being Catholic and not knowing about contraceptives increases the risk of not using them and so is the risk of catching sexual transmited diseases.

Keywords: Knowledge, family and contraceptives.

\section{RESUMO}

Introdução: O uso responsável de anticoncepcionais que nos permitam ter uma vida sexual saudável, depende do conhecimento que temos deles. Ferramentas e métodos: Estudo descritivo correlacional transversal com uma população de 477 universitários. Foi aplicado um questionário e analisado através da razão de prevalência, OR e IC95\% calculados por distribuição binomial com interações entre as variáveis SPSSV20. Resultados: Quanto à idade das participantes entre 17 e 27 anos, 54,7\% são mulheres, 75,3\% residem em Canar e 60\% são sexualmente ativas, das quais 92,7\% afirmaram saber sobre o uso de anticoncepcionais, 39,2\% não utiliza nenhum método de proteção. O método preferido é o Condon (32,5\%). Foi demonstrado que 16,1\% das alunas manifestaram não se responsabilizar por uma gravidez que se aproxima, não deseja. E destas $61,1 \%$ são do sexo feminino . Os alunos que não usam anticoncepcionais são católicos em 32\% (RP 1,32; IC95\% 1,12-2,63) e têm 50\% (RP 1,50; IC95\% 1,07-2,11) a mais de probabilidade de contrair doenças sexualmente transmissíveis. Aqueles com falta de conhecimento tem 2,4 vezes (RP 2,41; IC95\% 1,30-3,06) mais probabilidades de não usar nenhum método. Conclusões: $\mathrm{O}$ grau de conhecimento é alto, mas o uso de anticoncepcionais está muito aquém do esperado. Ser católica e não saber sobre anticoncepcionais aumenta o risco de não usá-los e também o risco de contrair doenças sexualmente transmissíveis.

Palavras-chave: Conhecimento, família e anticoncepcionais 


\section{Introducción}

La Organización Mundial de la Salud (OMS) describe que para el 2019 existían 1900 millones de mujeres en edad reproductiva, considerando a partir de los 15 años, de éstas 1112 millones necesitaban planificación familiar, una gran proporción utiliza métodos anticonceptivos (MAC), sin embargo, alrededor de 270 millones de mujeres no utilizan ningún MAC, a pesar de que a nivel mundial un gran porcentaje hace uso de MAC, existen países en los cuales la utilización de los mismos no llega al 50\%, lo que aumenta el riesgo de contraer no solo enfermedades de transmisión sexual (ETS), sino también, incrementa el riesgo de embarazos no deseados, y en el peor de los casos dichas infecciones durante el embarazo. (1-3)

El adecuado conocimiento del uso de métodos anticonceptivos en la población fértil tiene importancia en el ámbito preventivo, la asociación del conocimiento y el uso de un método seguro, evita el aumento de ETS, al igual que embarazos no planificados y abortos, considerando que la adolescencia en una etapa en la cual los comportamientos de riesgo son frecuentes al carecer de una adecuada información no solo sobre métodos anticonceptivos (MAC), sino también sobre sexualidad; muchos comportamientos son basados en las opiniones que pueden estar sujetas a mitos o criterios erróneos, mismos que son más aceptados por el círculo de amistades. (4-6)

La estructura familiar, las creencias culturales y religiosas pueden contribuir en la población joven con criterios errados sobre la salud sexual y reproductiva, en el sentido de que se puede ver a los métodos anticonceptivos, como una manera poco confiable en la relación de pareja y no se le considera como la manera más segura de tener contacto sexual. (6)

Es indispensable determinar un nivel de co- nocimiento de los métodos anticonceptivos que poseen los estudiantes de las carreras de salud y bienestar al igual que saber cuál es el método anticonceptivo considerado como más seguro y utilizado en esta población, lo que nos serviría como un punto de referencia para futuras investigaciones en la población joven en diferentes niveles de educación con el fin de ampliar la educación sexual a nivel académico y lograr la reducción de problemas relacionados con la deficiencia de conocimientos y uso de métodos anticonceptivos en estudiantes.

El presente estudio es un diseño observacional, descriptivo, correlacional de corte transversal, la población de estudio fueron los estudiantes de medicina y enfermería de la Universidad Católica de Cuenca sede Azogues, que accedieron a realizar la encuesta previa a su debida información. El análisis estadístico se realizó sobre la base de datos llamada "base_saludsexualyreproductiva". Para el análisis de los datos se utilizó el SPSS V.20.; Ios intervalos de confianza se calcularon en Gretl® 1 1.7.1, el gestor bibliográfico, Zotero para la bibliografía.

El análisis univariado se efectuó a través de los estimadores puntuales y sus intervalos de confianza. El análisis de las asociaciones entre variables categóricas, se realizó mediante el contraste de independencia a través de la razón de verosimilitudes, cálculo de Razón de Prevalencias (RP), Razón de Ventajas (OR), e Intervalos de Confianza del 95\% a partir de la distribución binomial.

\section{Resultados}

En esta investigación participaron 477 estudiantes entre 17 y 27 años de la Universidad Católica de Cuenca de la carrera de Medicina y Enfermería, de los cuales 261 (54,7\% IC95\% 49,47-58,49) fueron mujeres; del total de participantes 359 (75,3\%; IC95\% 71,06-78,83) residen en la provincia del Cañar, y 350 (73,4 IC95\% 76,94-68,97) estudiantes declararon ser católicos. En el 
gráfico 1 se muestra la distribución de la edad y sexo de los participantes, menores e iguales 22 años, 244 (51,2\% IC95\%
46,54-55,56) participantes tenían entre 18 y 22 años, como se muestra en el cuadro 1.

Gráfico 1. Distribución por edad y sexo de los participantes en el estudio

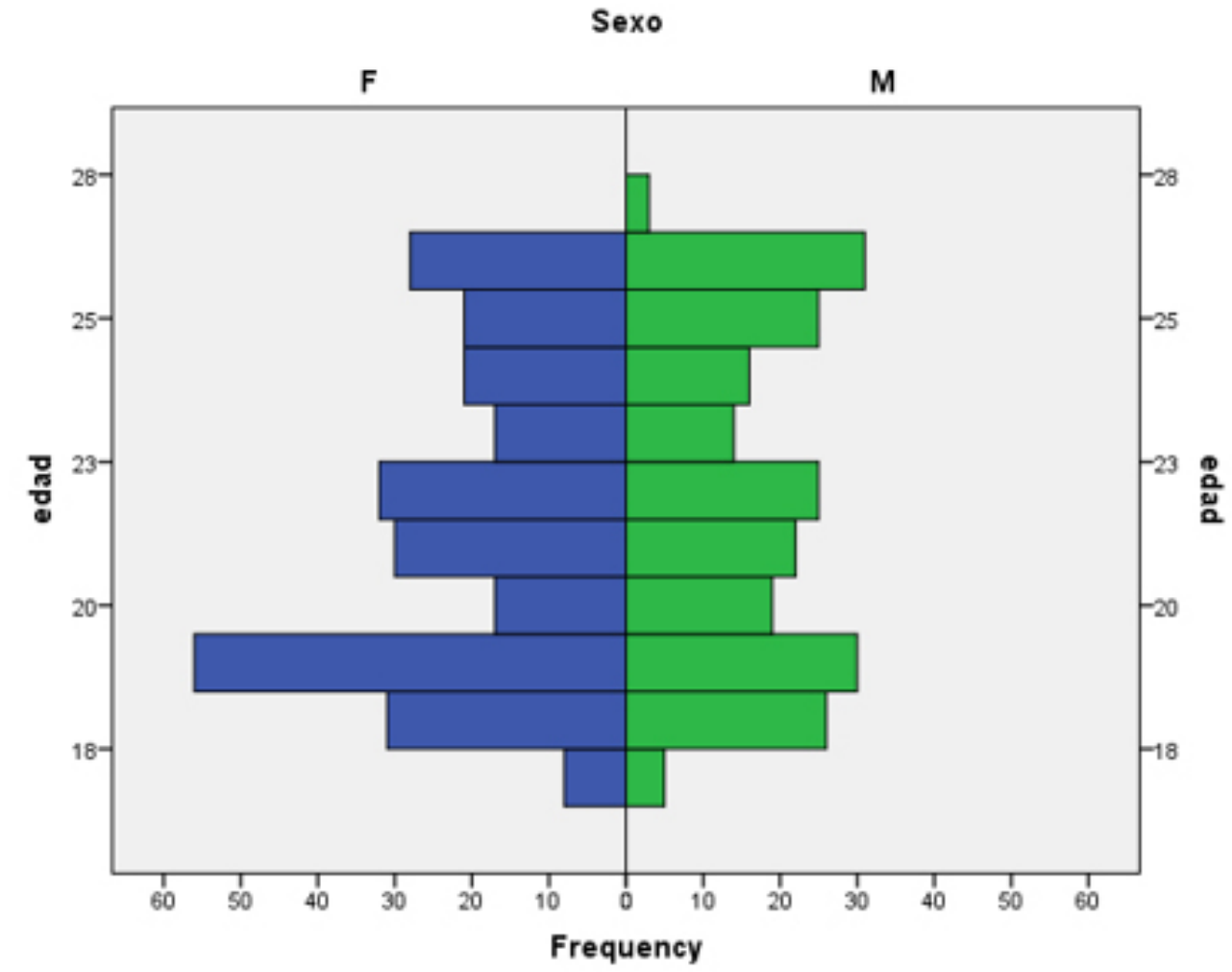

Autores: : Estudio "Conocimientos en salud sexual y reproductiva en estudiantes Universitarios, 2020"

Elaboración: Bravo S.

Tabla 1. Datos demográficos y creencias religiosas de los participantes

\begin{tabular}{|c|c|c|c|c|}
\hline Variable & Categoría & $\mathbf{N}$ & \% & IC95\% \\
\hline \multirow{3}{*}{ Sexo } & Hombre & 216 & 45,3 & $40,46-49,47$ \\
\cline { 2 - 5 } & Mujer & 261 & 54,7 & $49,47-58,49$ \\
\cline { 2 - 5 } & Total & $\mathbf{4 7 7}$ & $\mathbf{1 0 0 , 0}$ & \\
\hline \multirow{3}{*}{ Edad } & Menor de 22 años & 244 & 51,2 & $46,54-55,56$ \\
\cline { 2 - 5 } & Mayor o igual a 2 años & 233 & 48,8 & $43,61-52,41$ \\
\cline { 2 - 5 } & Total & $\mathbf{4 7 7}$ & $\mathbf{1 0 0 , 0}$ & \\
\hline \multirow{3}{*}{ Residencia } & Cañar & 359 & 75,3 & $71,06-78,83$ \\
\cline { 2 - 5 } & Otras provincias & 118 & 24,7 & $20,13-27,88$ \\
\cline { 2 - 5 } & Total & $\mathbf{4 7 7}$ & $\mathbf{1 0 0 , 0}$ & \\
\hline \multirow{3}{*}{ Religión } & Católica & 350 & 73,4 & $76,94-68,97$ \\
\cline { 2 - 5 } & Otras religiones & 127 & 26,6 & $22,01-29,98$ \\
\cline { 2 - 5 } & Total & $\mathbf{4 7 7}$ & $\mathbf{1 0 0 , 0}$ & \\
\cline { 2 - 5 } & & & \\
\hline
\end{tabular}

IC95\%: Intervalo de Confianza del 95\%

Autores: : Estudio "Conocimientos en salud sexual y reproductiva en estudiantes Univer- 
En cuanto a los datos demográficos y características de los participantes sexualmente activos se muestra en el cuadro 2 en donde 286 (60\%; IC95\% 55,5-64,3) manifestaron ésta condición, 151 (52,8\% IC95\%46,15$57,69)$ son mayores de 22 años, 220 (76,9\%; IC95\% 70,97-80,77) residen en la provincia del Cañar y 205 (71,7\% IC95\% 65,73-76,22) declararon ser católicos, en el gráfico 2 se muestra que entre los estudiantes que ya han mantenido relaciones sexuales la mediana de edad fue 22; la media fue de 21,9 años.

Tabla 2. Datos demográficos y creencias religiosas de los estudiantes sexualmente actiVOS

\begin{tabular}{|c|c|c|c|c|}
\hline Variable & Categoría & $\mathbf{N}$ & $\%$ & IC95\% \\
\hline \multirow{3}{*}{$\begin{array}{l}\text { Sexualmente } \\
\text { activos }\end{array}$} & $\mathrm{Si}$ & 286 & $60 \%$ & $55,50-64,35$ \\
\hline & No & 191 & $40 \%$ & $35,64-44,44$ \\
\hline & Total & 477 & 100,0 & \\
\hline \multirow{3}{*}{ Sexo } & Hombre & 139 & 48,6 & $42,30-53,85$ \\
\hline & Mujer & 147 & 51,4 & $45,10-56,54$ \\
\hline & Total & 286 & 100,0 & \\
\hline \multirow{3}{*}{ Edad } & Menor de 22 años & 135 & 47,2 & $41,26-52,80$ \\
\hline & Mayor o igual a 2 años & 151 & 52,8 & $46,15-57,69$ \\
\hline & Total & 286 & 100,0 & \\
\hline \multirow{3}{*}{ Residencia } & Cañar & 220 & 76,9 & $70,97-80,77$ \\
\hline & Otras provincias & 66 & 23,1 & $18,18-27,97$ \\
\hline & Total & 286 & 100,0 & \\
\hline \multirow{3}{*}{ Religión } & Católica & 205 & 71,7 & $65,73-76,22$ \\
\hline & Otras religiones & 81 & 28,3 & $22,72-33,22$ \\
\hline & Total & 286 & 100,0 & \\
\hline
\end{tabular}

IC95\%: Intervalo de Confianza del 95\%

Autores: : Estudio "Conocimientos en salud sexual y reproductiva en estudiantes Universitarios, 2020"

Elaboración: Bravo S. 


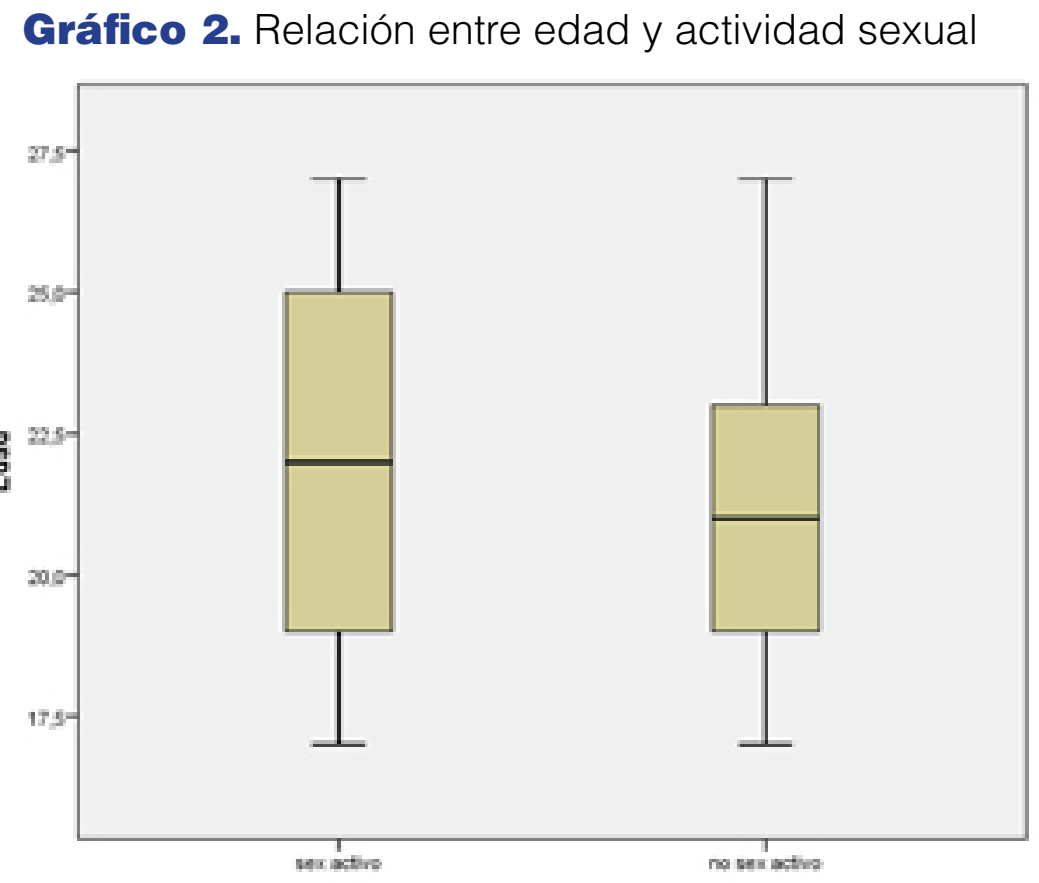

Participantes sexualmente activos

Autores: : Estudio "Conocimientos en salud sexual y reproductiva en estudiantes Universitarios, 2020"

Elaboración: Bravo S.

En lo que se refiere a la estructura familiar en el cuadro 3 se refleja que 177 (61,9\%; IC95\% 55,24-66,78) participantes manifestaron pertenecer a una familia nuclear clásica y 19 (6,6\%; IC95\% 3,49-8,74) ya formó su propia familia con su pareja actual, en el gráfico 3 se detalla la distribución de los diferentes tipos de familias nucleares, en donde el $67,6 \%$ corresponden a la clásica familia nuclear y un 30,5\% son familias que cuentan con un solo progenitor.

Cuadro 2. estructura familiar de los estudiantes sexualmente activos

\begin{tabular}{|l|l|c|c|c|}
\hline Variable & Categoría & $\mathbf{N}$ & $\mathbf{\%}^{\mathbf{a}}$ & $\mathbf{I C 9 5 \%}$ \\
\hline Tipo de familia & Nuclear clásica & 177 & 61,9 & $55,24-66,78$ \\
\hline & Monoparental & 80 & 28,0 & $22,73-33,22$ \\
\hline & Familia con padres ausentes & 5 & 1,7 & $0,35-3,49$ \\
\hline & Extendida & 3 & 1,0 & $0,34-2,45$ \\
\hline & Vive solo & 2 & 0,7 & $0,34-1,75$ \\
\hline & Ya formó su propia familia & 19 & 6,6 & $3,49-8,74$ \\
\hline & Total & $\mathbf{2 8 6}$ & $\mathbf{1 0 0 , 0}$ & \\
\hline
\end{tabular}

IC95\%: Intervalo de Confianza del 95\%

Autores: : Estudio "Conocimientos en salud sexual y reproductiva en estudiantes Universitarios, 2020"

Elaboración: Bravo S. 
Gráfico 3. Distribución de las familias nucleares

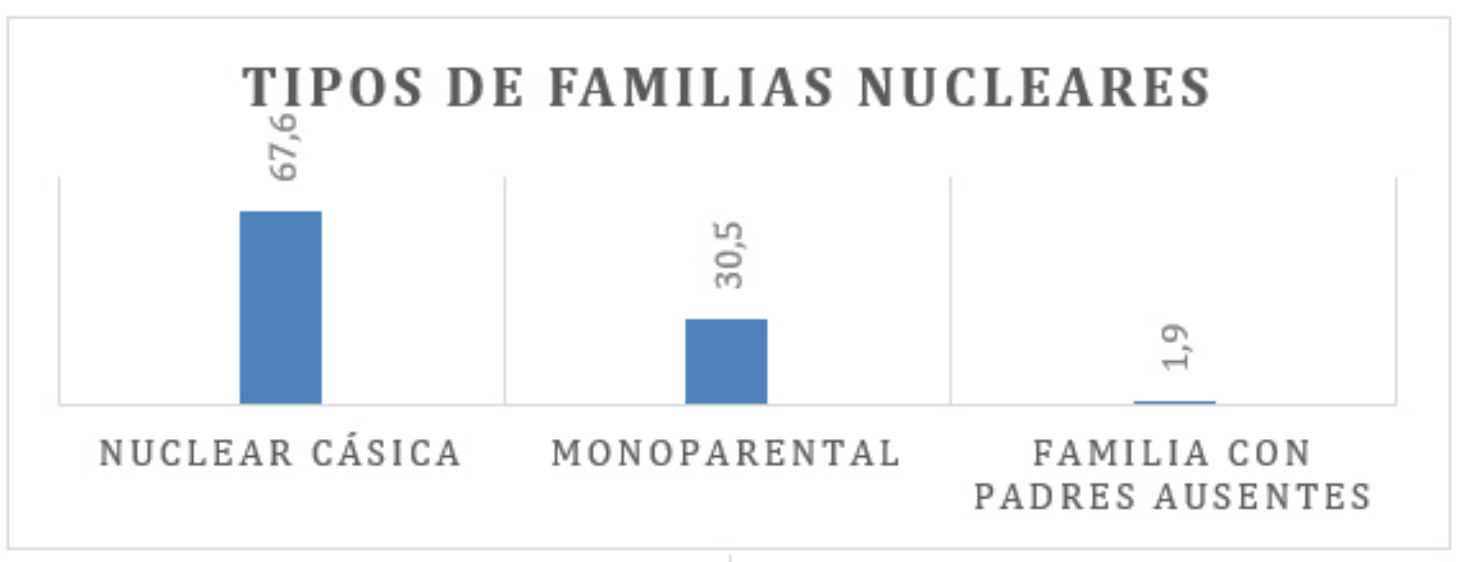

Autores: : Estudio "Conocimientos en salud sexual y reproductiva en estudiantes Universitarios, 2020"

Elaboración: Bravo S.

A pesar de que 265 (92,7\% IC95\% 88,81$95,10)$ estudiantes manifestaron conocer acerca de los métodos anticonceptivos, 112 (39,2 IC95\% 33,22-44,76) no utilizan ningún MAC y aquellos estudiantes que si lo hacen prefieren como mejor alternativa el preservativo, cuadro 3 y gráfico 4 . De los 112 estudiantes que manifestaron no utilizar ningún MAC al menos 28 (25\% IC95\% $16,96-30,04)$ estudiantes ya han tenido una
ETS, 25 (22,3\% IC95\% 14,29-29-46) ya tienen hijos, y la decisión que manifestaron tomar ante un embarazo no deseado, sólo 57 (50,9\% IC95\% 41,07-58,93) estudiantes asumirían su responsabilidad de forma inmediata, 18 (16,1\% IC95\% 9,82-23,21) no asume su responsabilidad y de éstos 11 $(61,1 \%$ IC95\% 38,89-83,33) corresponden al sexo femenino.

Cuadro 3. Métodos anticonceptivos más utilizados

\begin{tabular}{|l|l|c|c|c|}
\hline Variable & Categoría & N & \%a & IC95\% \\
\hline \multirow{4}{*}{ Conoce MAC } & $\mathrm{Si}$ & 265 & 92,7 & $88,81-95,10$ \\
\cline { 2 - 5 } & No & 21 & 7,3 & $4,19-10,14$ \\
\cline { 2 - 5 } & Total & $\mathbf{2 8 6}$ & $\mathbf{1 0 0 , 0}$ & \\
\hline \multirow{5}{*}{ Método anticonceptivo } & Preservativos & 93 & 32,5 & $26-57-37,41$ \\
\cline { 2 - 6 } & Inyectables & 26 & 9,1 & $5,94-12,89$ \\
\cline { 2 - 6 } & Tabletas & 7 & 2,4 & $0,69-3,85$ \\
\cline { 2 - 6 } & Combinan & 48 & 16,8 & $11,89-20,28$ \\
\cline { 2 - 6 } & No utilizan MAC & 112 & 39,2 & $33,22-44,76$ \\
\cline { 2 - 6 } & Total & $\mathbf{2 8 6}$ & $\mathbf{1 0 0 , 0}$ & \\
\hline \multirow{3}{*}{ ETS } & Si & 28 & 25 & $16,96-30,04$ \\
\cline { 2 - 6 } & No & 84 & 75 & $66,96-83,03$ \\
\cline { 2 - 6 } & Total & $\mathbf{1 1 2}$ & $\mathbf{1 0 0 , 0}$ & \\
\hline
\end{tabular}


CONOCIMIENTO Y USO DE MÉTODOS ANTICONCEPTIVOS EN ESTUDIANTES UNIVERSITARIOS DE MEDICINA Y ENFERMERÍA, 2020

\begin{tabular}{|l|l|c|c|c|}
\hline & Asume & 57 & 50,9 & $41,07-58,93$ \\
\cline { 2 - 5 } & Consejo familiar & 8 & 7,1 & $2,68-11,61$ \\
\cline { 2 - 5 } & Matrimonio & 29 & 25,9 & $16,96-33,04$ \\
\cline { 2 - 5 } & $\begin{array}{l}\text { No asume su responsa- } \\
\text { bilidad }\end{array}$ & 18 & 16,1 & $9,82-23,21$ \\
\cline { 2 - 5 } & Total & $\mathbf{1 1 2}$ & $\mathbf{1 0 0 , 0}$ & \\
\hline \multirow{2}{*}{$\begin{array}{l}\text { Sexo de estudián ante embarazo no deseado } \\
\text { responsabilidad }\end{array}$} & Mujer & 11 & 61,1 & $38,89-83,33$ \\
\cline { 2 - 5 } & Hombre & 7 & 38,9 & $16,67-61,11$ \\
\cline { 2 - 5 } & Total & $\mathbf{1 8}$ & $\mathbf{1 0 0 , 0}$ & \\
\hline
\end{tabular}

IC95\%: Intervalo de Confianza del 95\%

Autores: : Estudio "Conocimientos en salud sexual y reproductiva en estudiantes Universitarios, 2020"

Elaboración: Bravo S.

Gráfico 4. MAC utilizados por estudiantes universitarios

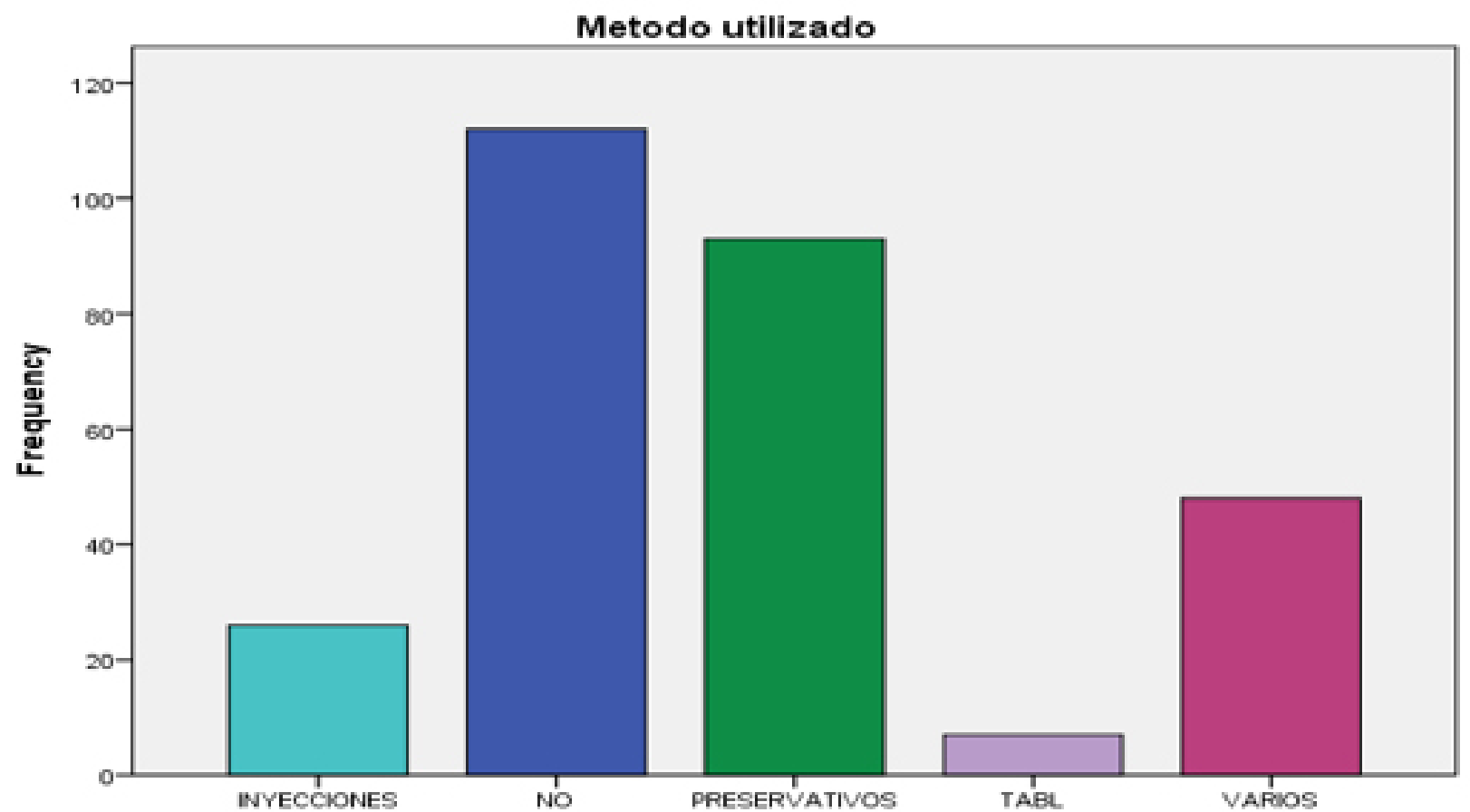

Autores: : Estudio "Conocimientos en salud sexual y reproductiva en estudiantes Universitarios, 2020"

Elaboración: Bravo S. 
El sexo, edad, lugar de residencia no tiene significancia en los estudiantes sexualmente activos para no usar MAC (RP 1,09; IC95\% 0,824-1,47), (RP 1,20; IC95\% 0,891,61), (RP 1,16; IC95\% 0,80-1,67) respectivamente, cuadro 4.

Los estudiantes sexualmente activos que manifestaron ser católicos tienen 32\% (RP 1,32; IC95\% 1,12-2,63) más probabilidad de no utilizar métodos anticonceptivos que aquellos de otra religión, mientras que la probabilidad de que los estudiantes que manifestaron no usar MAC contraigan alguna ETS fue 50\% (RP 1,50; IC95\% 1,07-2,11) mayor que los estudiantes que si utilizaron algún MAC, finalmente los estudiantes que no tienen conocimientos sobre MAC tienen 2,4 veces (RP 2,41; IC95\% 1,90-3,06) más probabilidad de no usar ningún MAC.

Cuadro 4. Características de los estudiantes sexualmente activos distribuidos por sexo, edad, residencia, religión

\begin{tabular}{|c|c|c|c|c|c|c|c|}
\hline Variable & Categorías & $\mathbf{N}$ & $\% a$ & $\mathbf{R P}$ & IC95\% & RV & Valor $p$ \\
\hline \multirow{3}{*}{ Sexo } & & & & 1,09 & $0,82-1,47$ & 0,38 & 0,53 \\
\hline & Hombre & 57 & 41,0 & & & & \\
\hline & Mujer & 55 & 37,4 & & & & \\
\hline \multirow{3}{*}{ Edad } & & & & 1,20 & $0,89-1,61$ & 1,39 & 0,23 \\
\hline & Menor de 22 años & 48 & 35,6 & & & & \\
\hline & Mayor o igual a 2 años & 64 & 42,4 & & & & \\
\hline \multirow{3}{*}{ Residencia } & & & & 1,16 & $0,80-1,67$ & 0,66 & 0,41 \\
\hline & Cañar & 89 & 40,5 & & & & \\
\hline & Otras provincias & 23 & 34,8 & & & & \\
\hline \multirow{3}{*}{ Religión } & & & & 1,32 & $1,12-2,63$ & 1,31 & 0,07 \\
\hline & Católica & 76 & 37,1 & & & & \\
\hline & Otras religiones & 36 & 44,4 & & & & \\
\hline \multirow{3}{*}{ ETS } & & & & 1,50 & $1,07-2,11$ & 7,83 & $<0,01$ \\
\hline & $\mathrm{Si}$ & 28 & 57,1 & & & & \\
\hline & No & 84 & 35,4 & & & & \\
\hline \multirow{3}{*}{ Conoce MAC } & & & & 2,41 & $1,90-3,06$ & 21,03 & $<0,01$ \\
\hline & $\mathrm{Si}$ & 94 & 35,5 & & & & \\
\hline & No & 18 & 85,7 & & & & \\
\hline
\end{tabular}

c El denominador para el porcentaje corresponde a la variable de la fila RP Razón de Prevalencia

IC95\% Intervalo de Confianza del 95\%

RV Razón de Verosimilitud

Autores: : Estudio "Conocimientos en salud sexual y reproductiva en estudiantes Universitarios, 2020"

Elaboración: Bravo S. 


\section{Discusión}

Mantener un buen nivel de conocimiento de los métodos anticonceptivos en la edad fértil permite a la persona la utilización responsable de los mismos y contribuyen a determinar la planificación familiar oportuna con la consecuente disminución de riesgos para la salud reproductiva, familiar y psicosocial, de aquí la importancia de determinar el nivel de conocimiento y el uso de los anticonceptivos en estudiantes universitarios en las áreas de salud y bienestar con el fin de establecer adecuadamente la problemática que conlleva el desconocimiento de los anticonceptivos en el mantenimiento de una vida sexual activa.

El inicio de la vida sexual cada vez es más temprana, así en la población de este estudio un $60 \%$ son sexualmente activos y se encuentran en el ciclo de vida de adolescencia tardía y adultos jóvenes, según manifiesta Rodríguez (5), que la población ha iniciado tempranamente la vida sexual siendo los adolescentes los que presentan mayores problemáticas relacionadas con embarazos no deseados y con aumento de la morbimortalidad.

Generalmente el conocimiento sobre anticonceptivos en la población fértil es buena y la adquieren de una manera empírica o a través de sus padres, profesores y amigos, sin embargo, existen los limitantes para determinar un uso adecuado de los mismos, siendo uno de los que refleja este estudio la condición religiosa que ha determinado el no usar un método anticonceptivo, lo que concuerda con el estudio realizado por Bojana Pinter et al, (2016) quienes manifiestan que las creencias culturales y religiosas influyen en la decisión de la utilización de un método anticonceptivo y a su vez está relacionado con la moralidad. (6)

Si bien es cierto el 92,7\% de los estudiantes conocen sobre MAC, un porcentaje considerable $(32,9 \%)$ no hace uso de los mis- mos, una realidad que se comparte a nivel mundial como se evidencian Kantorová et al, en la estimación de progresos de 185 países en donde el $24,2 \%$ no hacen uso de MAC, consideran como "necesidades desatendidas en materia de anticoncepción", así mismo II porcentaje de mujeres en edad reproductiva con las necesidades de planificación familiar satisfechas con métodos modernos era del $75,7 \%$ a nivel mundial en 2019, pero en zonas como África central y occidental esta cifra no llegaba al 50\%, lo cual indica una falla dependiente no solo de la calidad de información, sino también de las condiciones socioeconómicas. $(1,7)$

Esto conlleva a consecuencias nefastas como la adquisición ETS consideradas un problema mundial, causadas por más de 30 bacterias, de virus y parásitos diferentes, en latino América según la Organización Panamericana de la Salud (OPS), las personas sexualmente activas que padecen de una infección de trasmisión sexual son las de 15 a 49 años, sin embarga cada vez se observa que el inicio de la vida sexual es a edades más tempranas, como consecuencia la adquisición de ETS también están directamente relacionadas (8-10), en este estudio el $25 \%$ ha tenido ETS, situación que se asemeja a nuestro país vecino Colombia, $(11,12)$ en donde se evidencia que uno de cada cuatro adolescentes estudiantes ha tenido ETS, muchos relacionados al uso inadecuado de MAC, falta de uso de los mismos y al poco o inadecuado conocimiento sobre ETS. (13-15)

Otro aspecto a revisar son los embarazos como consecuencia de la falta de uso de MAC, al menos el 22,3\% ya tienen hijos, los cuales han asumido su función de padres y han formado ya su propia familia, sin embargo, existe un porcentaje del 16,1\% que manifestó no asumir su responsabilidad ante un embarazo no deseado a pesar de tratarse de una población universitaria del ámbito de Salud y Bienestar, pero lo más alarmante de éstos estudiantes es que más

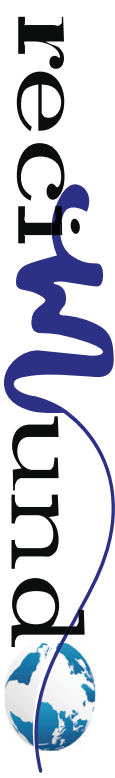


del $60 \%$ corresponden al sexo femenino.

A pesar de no haber encontrado evidencia con la cual comparar dichos datos, es importante mencionarlos, ya que esta población constituye aquella que en algún momento se enfrentará a dicha situación y al mantenerse con la opinión emitida podría constituir estudiantes que optarían por dejar a sus hijos con sus abuelos, darlos en adopción, abandonarlos y desentenderse de ellos o en el peor de los casos abortar, múltiples situaciones que hoy en día no son ajenos a nuestra realidad, mismos que se han evidenciado con una disminución al implementar el uso de MAC. (16)

Países como Honduras, Estados Unidos (EEUU) incluso Ecuador lograron disminuir los embarazos en adolescentes gracias a las estrategias intersectoriales en el periodo 2010-2013, logrando nuestro país un récord internacional al reducir en jóvenes de 10 a 14 años un 10,2\%, y en personas de 15 a 19 años de edad el 12,9\% de embarazos en adolescentes, el Ministerio de Salud Pública (MSP) emitió el Reglamento para regular el acceso y la disponibilidad de MAC, permitiendo el acceso a los mismos de forma gratuita y oportuna, incluida la anticoncepción oral de emergencia, acciones que han sido favorecedoras para esta población. (17)

En cuanto a los métodos anticonceptivos que mayor conocimiento tiene la población fértil del estudio es el anticonceptivo de barrera, básicamente el condón, siendo a su vez el el de preferencia para ser utilizado durante el acto sexual, sin embargo, según algunos estudios este método no es el único que se conoce existiendo otros MAC utilizados también por la población femenina, de acuerdo al Consenso Canadiense sobre anticoncepción según describe Amanda Negro et al, (2015) para la población femenina con mayor riesgo de presentar un embarazo no deseado establecen que el método de acción prolongada incluidos los implantes anticonceptivos y los dispositivos intrauterinos son métodos eficaces pero es- tán infrautilizados, por deficiencia en su conocimiento (18), por otro lado, según John K Amory (2016) manifiesta que el uso de condón se ha vuelto más predominante por mayor conocimiento y decisión masculina que son los que desean asumir la responsabilidad activa en la planificación familiar. (19)

Según la OMS el preservativo era considerado como el único MAC capaz de prevenir ETS incluido el VIH, así como embarazos (2), siempre y cuando el uso del mismo sea constante y de forma adecuada, sin embargo, se ha evidenciado en varios estudios no solo la adquisición de ETS, VIH, si no también embarazos ya sea por mal uso, fallas o rupturas durante la relación sexual como consecuencia de poder alcanzar un máximo placer sexual, siendo ésta una de las mayores preocupaciones del ser humano. $(14,20,21)$

La recopilación de información sexual en una población conservadora como la de nuestro estudio, generalmente puede representar ser perturbadora por lo que los datos obtenidos dependen mucho de la sinceridad de los participantes para la veracidad de los mismos, lo fundamental en nuestro medio radica en la prevención y promoción que se realice con la finalidad de disminuir los comportamientos sexuales de riesgo y aumentar el uso de forma adecuado de los MAC, de tal manera que los jóvenes pueden tener una vida sexual plena y placentera, sin riesgos ni consecuencias que puedan ser causa de acciones poco éticas.

\section{Conclusiones}

De los resultados obtenidos se puede concluir que los estudiantes de medicina y enfermería poseen un buen nivel de conocimientos sobre anticonceptivos, con un alto porcentaje sexualmente activos, sin embargo, aquellos que utilizan algún MAC se encuentran en un porcentaje inferior a lo esperado, siendo el más utilizado el preservativo 
y el que menos utilizan son las tabletas anticonceptivas.

Uno de los limitantes en el uso de los métodos anticonceptivos fue la religión siendo los católicos los que menos utilizan un MAC aumentando el riesgo de presentar una ETS, por lo que el uso adecuado de éstos aún sigue dependiendo de las condiciones culturales religiosas y de la estructura familiar de la persona.

Muchos estudiantes no asumen sus acciones responsablemente, lo que resulta controversial sobre todo en lo ético, ya que son estudiantes que al final de su carrera deberán ejercer su profesión en función a nuestro juramento hipocrático.

\section{Bibliografía}

1. Kantorová V, Wheldon MC, Ueffing P, Dasgupta ANZ. Estimating progress towards meeting women's contraceptive needs in 185 countries: A Bayesian hierarchical modelling study. PLOS Medicine. 18 de febrero de 2020;17(2):e1003026.

2. Organización Mundial de la Salud. Planificación familiar [Internet]. 2020 [citado 26 de agosto de 2020]. Disponible en: https://www.who.int/es/ news-room/fact-sheets/detail/family-planning-contraception

3. Organización Mundial de la Salud. Infecciones de transmisión sexual [Internet]. 2020 [citado 26 de agosto de 2020]. Disponible en: https://www.who. int/es/news-room/fact-sheets/detail/sexually-transmitted-infections-(stis)

4. Peláez Mendoza J. El uso de métodos anticonceptivos en la adolescencia. Revista Cubana de Obstetricia y Ginecología. marzo de 2016;42(1):0-0.

5. Rovalo BR. Factores asociados al inicio de vida sexual temprana en adolecentes en una unidad de medicina familiar de Querétaro. 13 de mayo de 2019 [citado 26 de agosto de 2020]; Disponible en: http://ri-ng.uaq.mx/handle/123456789/1486

6. Pinter B, Hakim M, Seidman DS, Kubba A, Kishen M, Di Carlo C. Religion and family planning. Eur $\mathrm{J}$ Contracept Reprod Health Care. diciembre de 2016;21(6):486-95.

7. Jiménez CAG, Díaz MH, Cardoso JJ. Riesgo preconcepcional y métodos anticonceptivos de control. Revisión bibliográfica. Gaceta Médica Espiri- tuana. 10 de abril de 2015;9(1):10.

8. Organización Mundial de la Salud. OMS | Infecciones de transmisión sexual [Internet]. WHO. World Health Organization; 2018 [citado 26 de agosto de 2020]. Disponible en: http://www.who.int/topics/ sexually_transmitted_infections/es/

9. Organización Panamericana de Salud. Infecciones de Transmisión Sexual - OPS/OMS | Organización Panamericana de la Salud [Internet]. 2019 [citado 26 de agosto de 2020]. Disponible en: http://www. paho.org/es/temas/infecciones-transmision-sexual

10. Ramirez MG, RiVERA EA, Torres ILR. CONOClMIENTO SOBRE PREVENCION DE INFECCIONES DE TRANSMISION SEXUAL EN ADOLESCENTES INMIGRANTES HABITANTES EN SOLEDAD-ATLANTICO 2018-2019. Identidad Bolivariana. 18 de febrero de 2020;4(1):5-15.

11. Villegas-Castaño A, Tamayo-Acevedo LS. Prevalencia de infecciones de transmisión sexual y factores de riesgo para la salud sexual de adolescentes escolarizados, Medellín, Colombia, 2013. latreia. 2016;29(1):5-17.

12. Von Sneidern E, Quijano L, Paredes MC, Obando E. PREVALENCIA DE ENFERMEDADES DETRANSMISION SEXUAL. 2016 [citado 26 de agosto de 2020];19(4). Disponible en: https://scholar. google.es/scholar?hl=es\&as_sdt=0\%2C5\&as_ $y l o=2015 \& q=P R E V A L E N C I A+D E+E N F E R M E D A-$ DES+DETRANSMISION+SEXUAL\&btnG=

13. Marco MHD, Ferraris $S$, Langsam M, Marco $M H D$, Ferraris $S$, Langsam M. Young population, sexually-transmitted diseases and rights. National and regional scenario in Argentina. Ciência \&amp; Saúde Coletiva. septiembre de 2018;23(9):283548.

14. Zelada Valdés A, Valdivia Romero Á, Saavedra Ramírez C, Oliva Pérez M, González Barroso M. Nivel de conocimientos sobre las Infecciones de Trasmisión Sexual (ITS) y conducta en adolescentes estudiantes de Enfermería. Revista Cubana de Medicina Tropical. agosto de 2014;66(2):295-304.

15. Torres PDNMP, Becerril PDMA, García SLL. Nivel de conocimiento sobre Infecciones de Transmisión Sexual (ITS)-VIH/SIDA en estudiantes de la Universidad Intercultural del Estado de Puebla. HOLOPRAXIS. 28 de junio de 2019;3(1):129-39.

16. Donoso S E, Vera P-G C. El aborto en Chile: aspectos epidemiológicos, históricos y legales. Revista chilena de obstetricia y ginecología. diciembre de 2016;81(6):534-45.

17. Ministerio de salud pública del Ecuador. Ecuador rompe récords internacionales en disminución del

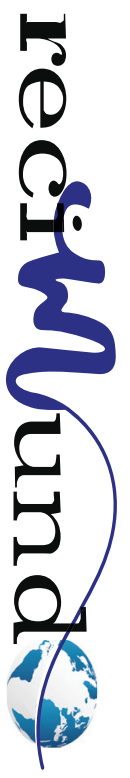


embarazo de adolescentes - Ministerio de Salud Pública [Internet]. 2020 [citado 26 de agosto de 2020]. Disponible en: https://www.salud.gob.ec/ ecuador-rompe-records-internacionales-en-disminucion-del-embarazo-de-adolescentes/

18. Black A, Guilbert E, Co-Authors, Costescu D, Dunn S, Fisher W, et al. Canadian Contraception Consensus (Part 1 of 4). J Obstet Gynaecol Can. octubre de 2015;37(10):936-42.

19. Amory JK. Male contraception. Fertility and Sterility. noviembre de 2016;106(6):1303-9. Disponible en: https://pubmed.ncbi.nlm.nih.gov/27678037/

20. Pérez Albitrez CM, Tirado Huamanchumo LC. Efi- cacia del taller educativo sobre el nivel de conocimiento del preservativo femenino en estudiantes universitarias, universidad privada antenor orrego, 2016. Universidad Privada Antenor Orrego [Internet]. 2019 [citado 26 de agosto de 2020]; Disponible en: http://repositorio.upao.edu.pe/handle/ upaorep/4615

21. Olmedo-Buenrostro BA, González-Robles HA, Delgado-Enciso I, Mora-Brambila AB, Cadenas Freixas JL, Montero Cruz SA. Frecuencia de infección por $\mathrm{VIH} /$ sida en usuarios de preservativo. Revista Cubana de Investigaciones Biomédicas. diciembre de 2015;31(4):480-9.

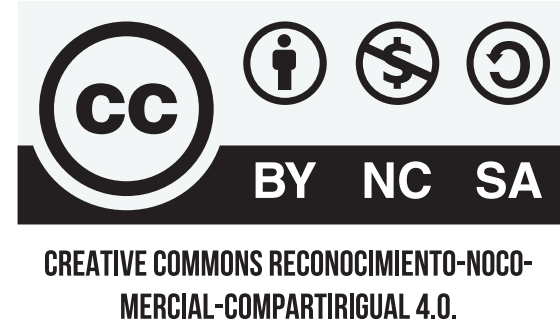

\section{CITAR ESTE ARTICULO:}

Bravo Salinas, S. E., Guerra Ortega, D. L., Uguña Rosas, V. A., \& Castillo Zhizhpón, A. A. (2020). Conocimiento y uso de métodos anticonceptivos en estudiantes universitarios de medicina y enfermería, 2020. RECIMUNDO, 4(4). https://doi.org/10.26820/ recimundo/4.(4).noviembre.2020.236-248 